\title{
El escrutinio diagnóstico limitado puede disminuir el impacto económico directo del síndrome de intestino irritable (SII)
}

\author{
Max J Schmulson \\ Limited diagnostic testing can \\ decrease the direct economic impact \\ of irritable bowel syndrome
}

Background: The economic impact of irritable bowel syndrome (IBS) in Mexico in terms of excessive diagnostic testing can be considerably reduced if the recommendations of the Latin American Consensus (LATAM) for IBS are followed. Aim: To estimate the economic impact of IBS in terms of excessive diagnostic testing. Material and Methods: Based on a previously published study, the costs of diagnostic testing for IBS were compared to the theoretical costs according to the recommendations of the consensus. These costs were compared to estimate the economic impact of excessive diagnostic testing. A costminimization analysis was also done. Results: For the lowest socioeconomic level in academic medicine, the excessive diagnostic testing had an approximate cost of US\$21.38, compared to US\$1.72 if the LATAM Consensus recommendations would have been followed, representing a saving of $92.0 \%$. The cost for the highest socioeconomic level in academic medicine was US\$1080.36 versus US $\$ 103.60$ (a saving of $90.4 \%$ ) and for private medicine, the costs were US\$3121.60 versus US\$159.90 (a saving of 94.9\%) if the recommendations would have been followed. Conclusions: Limited diagnostic testing recommended by the LATAM Consensus for IBS can significantly decrease the economic impact of this disease in Mexico (Rev Méd Chile 2008; 136: 1398-405).

(Key w ords: Diagnostic tests, routine; Direct service costs; Irritable bowel syndrome)

Recibido el 24 de marzo, 2008. Aceptado el 28 de julio, 2008.

Laboratorio de Hígado, Páncreas y Motilidad (HIPAM), Departamento de Medicina Experimental, Facultad de Medicina, Universidad Nacional Autónoma de México (UNAM).

$\mathrm{E}$ l síndrome de intestino irritable (SII) es uno de los trastornos de salud más frecuentes en el mundo. Su prevalencia varía de $10 \%$ a $20 \%$,

$\overline{\text { Correspondencia a: Max Schmulson W. Laboratorio de Hígado, }}$ Páncreas y Motilidad (HIPAM), Departamento de Medicina Experimental, Hospital General de México. Dr. Balmis \#148 Col. Doctores C.P.06726. México D.F.-México. Tel: 52-5556232673. Fax: 52-5556232669. E mail: maxjulio@avantel.net siendo más común en mujeres, entre los 30 y 50 años $^{1-2}$. En Latinoamérica se considera que tiene una prevalencia de $9 \%$ a $18 \% 3,4$. Además, es el primer motivo de consulta al gastroenterólogo y está entre los diagnósticos más comunes en medicina general ${ }^{5}$.

El SII representa un alto impacto económico en costos directos (relacionados con el sistema de salud, diagnóstico, consultas médicas, tratamien- 
to) e indirectos (ausentismo, disminución en la productividad laboral) ${ }^{5-7}$. En Estados Unidos de Norteamérica se han reportado costos directos de $\$ 1,6$ mil millones e indirectos de $\$ 19,2$ mil millones de dólares anuales ${ }^{8}$. En Latinoamérica no existen estudios que evalúen el impacto económico del SII. Un estudio en México demostró que a pesar de los reconocidos criterios diagnósticos de Roma para SII y las recomendaciones sobre el uso limitado de pruebas diagnósticas, se solicitan numerosos estudios de laboratorio e imagen en dichos pacientes, pero se desconoce a ciencia cierta el impacto económico de éstos ${ }^{9}$.

En el año 2004 se publicó el Consenso Latinoamericano (LATAM) sobre el SII, planteando el abordaje diagnóstico y tratamiento de este trastorno en nuestra región ${ }^{3,10}$. Según este Consenso, el escrutinio diagnóstico debe limitarse a ciertas pruebas de laboratorio (Tabla 1) y se debe realizar colonoscopia ante signos de alarma (pérdida de peso, hemorragia, anemia, fiebre, historia familiar de cáncer de colon o enfermedad inflamatoria intestinal), inicio de síntomas $>50$ años o cambio en el patrón de los síntomas ${ }^{10}$. Sólo se recomienda evaluación para enfermedad celíaca en regiones con una prevalencia mayor a $1 \%{ }^{11}$.

Nuestra hipótesis es que se puede lograr un ahorro económico importante en la evaluación de pacientes con SII, si los médicos y proveedores de servicios de salud seguimos las recomendaciones de los diversos consensos. Por lo anterior, nuestro objetivo fue estimar el impacto económico del SII en términos de estudios diagnósticos excesivos de acuerdo a los datos del estudio inicial publicado en México (estudio inicial) ${ }^{9}$, y compararlo con el costo teórico aplicando las recomendaciones del Consenso LATAM.

\section{MATERIAL Y MÉTODOS}

El estudio inicial ${ }^{9}$ analizó 98 pacientes con SII según los criterios de Roma ${ }^{12}$ y sin comorbilidad conocida del Instituto Nacional de Ciencias Médicas y Nutrición Salvador Zubirán (INCMNSZ), un centro de referencia de pacientes de la República Mexicana. Estos pacientes fueron seguidos por 33,4 meses (2,78 años) durante los cuales se solicitó un promedio de 22,4 pruebas por paciente (Tabla 2).

Para evaluar el impacto económico de estos exámenes excesivos, se compararon los costos con los gastos teóricos de acuerdo a las recomendaciones del Consenso LATAM. Se obtuvieron los costos de cada prueba de acuerdo a la mínima y máxima clasificación socioeconómica en el año 2004 en el INCMNSZ y en medicina privada. En el INCMNSZ existen siete niveles de clasificación; en el nivel 1 se subsidia 98\% y el paciente paga únicamente $2 \%$, en el nivel 7 el paciente paga $100 \%$. Para fines de este estudio se compararon solamente los datos para nivel 1 y 7 . Los costos en medicina privada para el mismo período de tiempo se obtuvieron de la Clínica Lomas Altas, un centro de atención ambulatoria del poniente de la Ciudad de México, área de población con el mayor nivel socioeconómico de la ciudad.

Para determinar el costo total de las pruebas con base en el estudio inicial, se utilizó la siguiente fórmula: $\sum$ ([mediana de cada prueba $\div$ 2,78]) $\mathrm{x}$ costo de la prueba).

En esta, la mediana de cada prueba se dividió entre 2,78 (años de seguimiento) para calcular el número anual de exámenes y luego se multiplicó por el costo de cada prueba. Luego se sumaron los resultados obtenidos para cada una de las

\title{
Tabla 1. Exámenes para el escrutinio diagnóstico limitado sugeridos por el Consenso Latinoamericano (LAT AM ) sobre el SII
}

\author{
Hemograma \\ Perfil bioquímico \\ Pruebas de función tiroidea \\ Sangre oculta y parásitos en heces \\ Pruebas para enfermedad celíaca en países con alta prevalencia
}

Modificado de Schmulson M y col. Gastroenterol Hepatol 2004; 27: 635-6. 
pruebas descritas en el Tabla 2 y así se obtuvo el costo total de la evaluación diagnóstica por año de los pacientes con SII en cada uno de los niveles descritos.

También se determinaron los costos teóricos de los exámenes según las recomendaciones del Consenso LATAM, los cuales incluyen: hemograma, sedimentación globular, perfil bioquímico, perfil tiroideo, coproparasitológico y hemorragia oculta en deposiciones, y colonoscopia en mayores de 50 años o con signos de alarma. Para estimar el ahorro económico potencial, los costos calculados del estudio inicial para cada uno de los niveles, fueron comparados con los costos teóricos utilizando las recomendaciones del Consenso.
Los costos se expresan en dólares americanos de acuerdo al tipo de cambio del año 2004 y el potencial ahorro, en porcentajes.

Además se realizó un análisis de minimización de costos, dividiendo los costos totales según el estudio inicial y según las recomendaciones del Consenso LATAM, sobre el tiempo. Se asumió como tiempo 12 meses para ambos casos, ya que lo más probable es que una vez que se apliquen los lineamientos del Consenso, no se soliciten más exámenes al menos durante 1 año. Se eligió este tipo de análisis ya que siguiendo o no las recomendaciones del Consenso LATAM, se debe llegar al mismo diagnóstico y al mismo resultado en el paciente ${ }^{13}$.

\section{Tabla 2. Frecuencia de estudios de laboratorio y gabinete practicados a los pacientes con SII*}

\begin{tabular}{|lc|}
\hline Prueba & M ediana/Año \\
\hline Hemograma & 1,97 \\
Velocidad de sedimentación & 0,35 \\
Coproparasitológico & 1,44 \\
Hemorragia oculta en deposiciones & 1,08 \\
Perfil bioquímico 1 & 3,41 \\
Orina completa & 2,6 \\
Urocultivo & 1,25 \\
Perfil lipídico & 3,24 \\
Electrolitos plasmáticos & 1,08 \\
Pruebas de coagulación & 0,72 \\
Perfil tiroideo & 0,89 \\
Pruebas de malabsorción 2 & 1,08 \\
Rectosigmoidoscopia flexible & 0,54 \\
Enema baritado & 0,72 \\
Colonoscopia & 0,18 \\
Radiografía baritada de esófago, estómago y duodeno & 0,72 \\
Endoscopia digestiva alta & 0,54 \\
Tránsito intestinal & 0,18 \\
Ecotomografía abdominal & 0,54 \\
Electrocardiograma & 0,72 \\
Radiografía de tórax & 1,08 \\
\hline
\end{tabular}

*Modificado de la referencia 9.

${ }^{1}$ Incluyó glicemia, nitrógeno ureico, creatininemia, aminotransferasas, fosfatasas alcalinas, bilirrubinemia.

2Incluyeron carotenos, grasa en heces, anticuerpos antigliadinas y antiendomisio, pruebas de D-xylosa y de dilaurato de fluoresceína. 


\section{REsultados}

Los exámenes excesivos tienen un costo aproximado de US\$21,38 dólares al año para el nivel 1 de medicina institucional y US\$1.080,36 para el nivel 7. En medicina privada, el costo es de US\$3.121,60. En contraste, siguiendo las recomendaciones del Consenso LATAM, el costo estimado de las pruebas diagnósticas sería de US\$1,72 y US\$103,60 en los dos niveles de medicina institucional y de
US\$159,90 en medicina privada. El potencial ahorro sería de 92,0\% para el nivel 1 y 90,4\% para el nivel 7 y de $94,9 \%$ en medicina privada (Figura 1). Si se requiere colonoscopia, los costos según el Consenso serían de US\$7,00 y US\$363,73 en medicina institucional y $\$ 978.18$ dólares en medicina privada. El ahorro sería de $59,2 \%, 56,7 \%$ y $63,5 \%$, respectivamente (Figura 2). Así mismo, el análisis de minimización de costos también muestra una diferencia importante (Tabla 3).

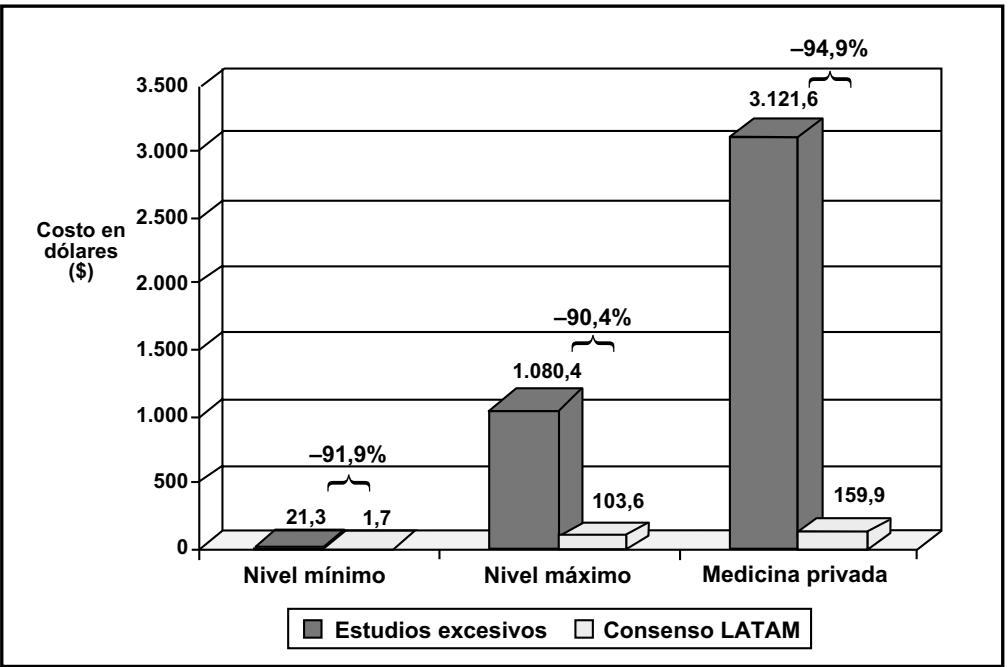

Figura 1. Pruebas de diagnóstico excesivas comparadas con las recomendaciones del consenso LATAM.

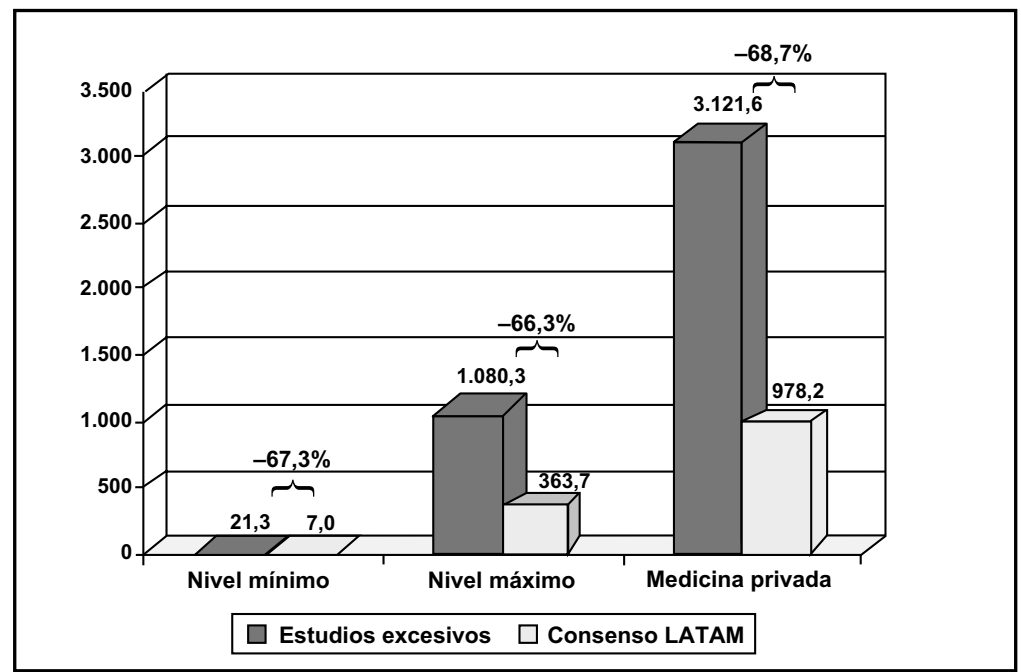

Figura 2. Pruebas de diagnóstico excesivas comparadas con las recomendaciones del Consenso LATAM incluyendo colonoscopia cuando esté indicada. 
Tabla 3. Análisis de minimización de costos por año*

\begin{tabular}{|lccc|}
\hline Nivel de Atención & Estudios excesivos & $\begin{array}{c}\text { Lineamientos del } \\
\text { Consenso LATAM }\end{array}$ & $\begin{array}{r}\text { Lineamientos del } \\
\text { Consenso LATAM con } \\
\text { colonoscopia }\end{array}$ \\
\hline $\begin{array}{l}\text { Nivel más bajo Institución } \\
\text { Nivel más alto }\end{array}$ & 1,78 & 0,24 & 0,58 \\
Institución & 90,03 & 8,63 & 30,30 \\
Medicina privada & 260,13 & 13,32 & 81,51 \\
\hline
\end{tabular}

*Calculadas por conversión a dólares americanos.

\section{DiscUSIÓN}

Este es el primer estudio que analiza el impacto económico del SII en Latinoamérica en términos de exámenes diagnósticos excesivos. Basado en un estudio previo en México, hemos encontrado que el costo por año en estudios de laboratorio, imagen y endoscopia, va de 21 hasta 1.080 dólares en medicina institucional, hasta 3.121 dólares en medicina privada y que si se utilizan las recomendaciones del Consenso LATAM sobre el SII 3,10 este ahorro puede llegar a ser de $66 \%$ a $90 \%$. Además, resulta de costo/utilidad aplicar los lineamientos de dicho Consenso. Lo anterior indica un impacto económico muy alto en una enfermedad funcional que no tiene ninguna alteración orgánica o estructural que se pueda reflejar en exámenes diagnósticos.

Nosotros sólo determinamos el costo anual en términos de estudios de laboratorio e imagen, pero nuestros resultados son similares a los de otros investigadores que han reportado altos costos económicos en SII en comparación con otras enfermedades. Por ejemplo, Longstreth y cols $^{14}$ compararon los costos directos en pacientes con y sin SII encontrando que tanto en estudios radiológicos como de laboratorio, los costos fueron significativamente más altos en los primeros. Los costos directos, incluyendo consultas, hospitalizaciones y prescripciones fueron $51 \%$ más altos en SII que en pacientes sin SII. Otro estudio realizado por Talley y cols ${ }^{15}$ en población abierta, comparó los costos de consultas médicas, laboratorios, radiología, emergencias y hospitalizaciones durante un año, encontrando que los pacientes con SII representaban costos de 742 dólares comparado con 429 en controles. Al ajustar por edad, sexo, educación, estado civil y trabajo, los gastos directos por la atención médica fueron 1,6 veces mayores en SII que en los controles. Es de anotar que esta población es comparable con el nivel alto de medicina institucional de nuestro estudio.

Bentkover y cols ${ }^{16}$ estimaron los costos anuales de pacientes con SII en Canadá, incluyendo honorarios médicos, pruebas diagnósticas, medicamentos, referencia a especialistas y emergencias. Encontraron que el costo anual promedio por paciente con SII era de 154 a 160 dólares.

Está bien demostrado que las pruebas diagnósticas proporcionan muy poca información adicional en pacientes que cumplen criterios de Roma para SII y que no tienen síntomas de alarma ${ }^{17}$. En el estudio inicial en México ${ }^{9}$ encontramos que aunque el diagnóstico de SII se estableció en la primera consulta en $87 \%$ de los casos, se solicitaron en promedio 17,4 estudios después del diagnóstico. A pesar de que el diagnóstico se establece basado en criterios clínicos durante la primera consulta en la mayoría, se siguen solicitando exámenes excesivos, probablemente porque para el médico no siempre es fácil establecer el diagnóstico de SII. Por un lado, el SII no tiene un marcador biológico universal, lo que hace que el diagnóstico sea con base en síntomas (criterios), que muchas veces son difíciles de valorar objetivamente $^{18}$. Por otra parte, el propio médico siente inseguridad en su diagnóstico en ausencia de datos objetivos, lo cual ha sido previamente descrito para SII y dispepsia funcional, en compa- 
ración con la seguridad que sienten sobre sus diagnósticos de enfermedades orgánicas ${ }^{19}$. Tomando en cuenta que el SII no es un diagnóstico de exclusión, Spiegel y cols ${ }^{20}$ evaluaron la opinión de enfermeras, médicos generales, gastroenterólogos y expertos en SII. Encontraron que $42 \%$ de los gastroenterólogos y $76 \%$ de enfermeras y médicos generales creían que el diagnóstico de SII era de exclusión, en comparación con solo $8 \%$ de los expertos. Estos últimos solicitaron significativamente menos pruebas que el resto de participantes, lo cual se tradujo en menores costos, US\$297 contra US\$658 en los no expertos. En el estudio inicial mexicano, muchas veces los pacientes fueron manejados en la consulta de medicina interna y no necesariamente fueron referidos a gastroenterología, sugiriendo que el grado de especialización y conocimiento de los médicos sobre SII, puede explicar en parte el número excesivo de pruebas ordenadas que se traduce en altos costos en el diagnóstico como demostramos en el presente estudio.

Puede quedar la incertidumbre para el médico y el paciente de que los síntomas de SII estén enmascarando un padecimiento orgánico importante $^{20}$. Esto ya ha sido descartado por Cash y cols $^{17}$ quienes demostraron que en SII sin síntomas de alarma, es muy baja la probabilidad preprueba de que los pacientes padezcan padecimientos como enfermedad intestinal inflamatoria, cáncer de colon o infección intestinal, comparables con la prevalencia de estas enfermedades en la población general.

Aunque la colonoscopia no está indicada en pacientes con SII menores de 50 años a menos que existan síntomas de alarma ${ }^{2,10,21-22}$ muchas veces se realiza por inquietud tanto del médico como del paciente. Con la hipótesis de que la información de una colonoscopia negativa puede mejorar la calidad de vida al tranquilizar al paciente con SII, Spiegel y cols $^{23}$ realizaron un estudio en 458 pacientes con SII a quienes se les aplicó un cuestionario y se relacionó con el antecedente de colonoscopia, no encontrando asociación entre una colonoscopia negativa y la tranquilidad del paciente o una mejor calidad de vida $^{23}$. Nosotros hemos demostrado que aun en el caso de que esté indicado solicitar colonoscopia, el ahorro económico sería mayor de $60 \%$ según las recomendaciones del Consenso LATAM $^{3,10}$.
Otro punto importante es que los pacientes con SII pueden presentar padecimientos somáticos y físicos relacionados con su problema psicosocial, que puede interpretarse como una condición orgánica subyacente. Se ha encontrado que existe una relación entre el grado de somatización y el número de pruebas diagnósticas solicitadas en SII, considerando que la somatización puede llevar al médico a pedir más exáme$n^{2}{ }^{24}$. Por ello se recomienda que el médico esté vigilante de la somatización, para poder referir a estos pacientes ${ }^{18}$. Finalmente hay que mencionar que existen datos suficientes que han demostrado que bien aplicados los criterios de Roma para SII, la probabilidad de excluir un diagnóstico orgánico o maligno es menor de $3 \% 25-27$. Por ello, los diferentes grupos de expertos y consensos como el LATAM ${ }^{3,11}$ han establecido lineamientos sobre el uso limitado de pruebas diagnósticas para evitar un alto costo en el abordaje diagnóstico y reducir el impacto económico directo del SII.

El análisis de los exámenes excesivos utilizados en nuestro estudio, se realizó con pacientes SIIRoma ${ }^{12}$ ya que los criterios de Roma II $^{28}$ no se habían traducido y los de Roma III $^{29}$ no estaban aún disponibles. Sin embargo los nuevos criterios no invalidan las investigaciones realizadas con pacientes diagnosticados mediante criterios previos ni debe ser el factor por el cual se cambie el diagnóstico de los pacientes en la clínica. Además los datos derivados de investigaciones realizadas con criterios anteriores, siguen siendo la base fundamental para continuar modificando los criterios $^{12,28-29}$. No consideramos entonces que el utilizar pacientes con SII-Roma III modificaría nuestros resultados, ya que los costos excesivos dependen principalmente de la falta de conocimiento que tienen los médicos sobre el diagnóstico basado en criterios y un escrutinio limitado. Más aún, en países de lengua no inglesa, los criterios son emitidos con un retraso dependiente del proceso de traducción y validación al idioma y cultura en cuestión. Esto es de vital importancia y Gerson y cols, en un estudio realizado en Estados Unidos de Norteamérica, México, Canadá, Inglaterra, Italia, Israel, India y China, encontraron diferencias significativas en los síntomas del SII, sugiriendo que pueden estar determinadas por creencias culturales, localización urbana o rural, o costumbres dietéticas $^{30}$. Por lo anterior Drossman y Weinland 
insisten en su editorial que los cuestionarios y criterios diagnósticos deben estandarizarse y traducirse con métodos estrictos y adecuada validación intercultural para tener la certeza que se están evaluando grupos similares en términos de síntomas $^{31}$. Si bien los criterios de Roma III fueron publicados en 2006, aún no están disponibles en español, lo cual se encuentra en un proceso conjunto en México, Chile, Nicaragua, Honduras, Guatemala, España y Carolina del Norte en Estados Unidos de Norteamérica, patrocinado por la Fundación de Roma.

Una limitante del presente estudio es que se trata de un análisis teórico basado en un estudio que había analizado, retrospectivamente, el número de exámenes que se solicitan en un centro de referencia para pacientes con SII y se ha comparado con el número que se debe solicitar de acuerdo a un Consenso. Sin embargo, en un estudio prospectivo, es probable que los médicos reduzcan el número de exámenes solicitados por el hecho de conocer que están participando precisamente en una investigación que trata de determinar el costo económico derivado de solicitar pruebas excesivas. Además hemos asumido que en medicina privada se solicita el mismo número de estudios que en medicina institucional,

\section{REFERENCIAS}

1. AGA Technical Review on Irritable Bowel Syndrome. Gastroenterology 2002; 123: 2108-31.

2. American College of Gastroenterology Functional Gastrointestinal Disorders Task Force. EvidenceBased Position Statement on the Management of Irritable Bowel Syndrome in North America. Am J Gastroenterol 2002; 97: S1-S5.

3. Valenzueia J, Alvarado J, Cohen H, Damiao A, FrancisCONI C, Frugone L et aL. Un consenso latinoamericano sobre el síndrome de intestino irritable. Gastroenterol Hepatol 2004; 27: 325-43.

4. López-Colombo A, Bravo-Gonzáles D, Corona-López A, Pérez-López ME, Cervantes-Ocampo M, Romero-Ogawa ET AL. First community-based study of functional gastrointestinal disorders (FGID) in Mexico using the Rome II modular questionnaire. (Abstract) Gastroenterology 2006; 130(Suppl 2): A508.

5. Russo M, Gaynes B, Drossman D. A national survey of practice patterns of gastroenterologists with comparison to the past two decades. J Clin Gastroenterol 1999; 29: 339-43. lo cual no hemos comprobado pero la experiencia clínica así nos lo indica. Finalmente, no hemos analizado si el escrutinio diagnóstico limitado sugerido, redundaría en un mejor control de los síntomas del SII.

En conclusión, el presente estudio proporciona información sobre una parte de los costos directos que representa el SII en México, demostrando que el escrutinio diagnóstico limitado recomendado por el Consenso LATAM sobre el SII puede disminuir el impacto económico del mismo. Se requieren estudios para establecer los costos totales en México y Latinoamérica, tomando en cuenta las consultas médicas, hospitalizaciones, emergencias y medicamentos y poder cuantificar el impacto económico directo del SII.

\section{Agradecimientos}

Al Dr. Juan Manuel Mejía-Arangure, Unidad de Epidemiología Clínica, Hospital de Pediatría, Centro Médico Nacional Siglo XXI, Ciudad de México, por sus recomendaciones en el análisis de los datos. Los datos de este trabajo fueron presentados en forma de resumen durante el Congreso Mundial de Gastroenterología 2005 en Montreal-Canadá y durante la Semana Nacional de Gastroenterología en México DF 200527-28.

6. MARTIN R, BARRON JJ, ZACHER C. Irritable bowel syndrome: Toward a cost-effective management approach. Am J Manag Care 2001; 7: S268-S275.

7. Cash B, Suluvan S, Barghout V. Total costs of IBS: Employer and managed care perspective. Am J Manag Care 2005; 11: S7-S16.

8. Sandier RS, Everhart JE, Donowitz M, Adams E, Cronin $\mathrm{K}$, GoOdman $\mathrm{C}$ ET AL. The burden of selected digestive diseases in the United States. Gastroenterology 2002; 122: 1500-11.

9. Schmulson M, VaLdovinos MA. Utilización de recursos médicos por los pacientes con síndrome de intestino irritable en un hospital de tercer nivel. Rev Gastroenterol Mex 1998; 63: 6-10.

10. Schmulson M, Valenzuela J, Alvarado J, Cohen H, Damao A, Francisconi C et al. Cartas al director: Consenso latinoamericano sobre el síndrome del intestino irritable: algoritmo de diagnóstico y tratamiento. Gastroenterol Hepatol 2004; 27: 635-6.

11. Spiegel BM, DeRosa VP, Gralnek IM, Wang V, Dulai GS. Testing for celiac sprue in irritable bowel syndrome with predominant diarrhea: A cost-effectiveness analysis Gastroenterology 2004; 126: 1721-32. 
12. THOMPSON WG AND THE WORKING TEAM FOR FUNCTIONAL. BOWEL DISORDERS. C. Functional bowel disorders and D. Functional abdominal pain. En: Drossman DA, Richter JE, Talley NJ, Thompson WG, Corazziari E, Whitehead WE. Editores. The functional gastrointestinal disorders. Boston: Little Brown and Company; 1994; p. 115-73.

13. Sackett DL, Haynes RB, GuyatT GH, Tugwell P. How to read reviews and economic analyses. En: Sackett DL, Haynes RB, Guyatt GH, Tugwell P. Editores. Clinical epidemiology, A basic science for clinical medicine. Boston: Little Brown and Co Inc; 1991; p. 379-392.

14. Longstreth GF, Wilson A, Knight K, Wong J, Chiou $\mathrm{CF}$, BaRGHOUt $\mathrm{V}$ ET AL. Irritable bowel syndrome: health care use and costs: A U.S. managed care perspective. Am J Gasroenterol 2003; 98: 600-7.

15. Tauey NJ, Gabriel SE, Harmsen WS, Zinsmeister AR, Evans RW. Medical costs in community subjects with imitable bowel syndrome. Gastroenterology 1995; 109: 1736-41.

16. Bentkover Jd, Field C, Greene EM, Plourde V, CasciaNo JP. The economic burden of irritable bowel syndrome in Canada. Can J Gastroenterol 1999; 13(Suppl A): 89A-96A.

17. Cash B, Schoenfeid P, Chey W. The utility of diagnostic tests in irritable bowel syndrome patients: A systematic review. Am J Gastroenterol 2002; 97: 2812-19.

18. SPIEGEL BM. Do physicians follow evidence-based guidelines in the diagnostic work-up of IBS? Nature Clin Pract Gastroenterol Hepatol 2007; 4: 296-7.

19. Crean GP, Holden RJ, Knil-Jones RP, Beattie AD, James WB, Marjoribanks FM. A database on dyspepsia. Gut 1994; 35: 191-202.

20. Spiegel BM, Farid M, Esrailan E, Taley J, Chang L. Is irritable bowel syndrome a diagnosis of exclusion? A survey of primary care providers, gastroenterologists, and IBS experts. (Abstract). Gastroenterology (Suppl S2) 2006; 130: A111.

21. Drossman DA. Irritable bowel syndrome: How far do you go in the workup? Gastroenterology 2001; 121: $1512-15$.
22. Drossman DA, Whitehead WE, Camileri M. Irritable bowel syndrome: A technical review for practice guideline development. Gastroenterology 1997; 112: 2120-37.

23. Spiegel BM, Gralnek im, Bolus R, Chang L, Dulai GS, NALiboff B, MaYer EA. Is a negative colonoscopy associated with reassurance or improved healthrelated quality of life in irritable bowel syndrome? Gastrointest Endosc 2005; 62: 892-9.

24. Spiegel BM, Kanwal F, Naliboff B, Mayer E. The impact of somatization on the use of gastrointestinal health-care resources in patients with irritable bowel syndrome. Am J Gastroenterol 2005; 100: 2262-73.

25. Owens DM, Nelson DK, TALey NJ. The irritable bowel syndrome: long-term prognosis and the physician-patient interaction. Ann Intern Med 1995; 122: 107-12.

26. García Rodríguez LA, Ruigomez A, Wallander MA, Johansson S, Olbe L Detection of colorectal tumor and inflammatory bowel disease during follow-up of patients with initial diagnosis of irritable bowel syndrome. Scand J Gastroenterol 2000; 35: 306-11.

27. Thompson WG, Heaton KW, Smyth GT, Smyth C. Irritable bowel syndrome in general practice: prevalence, characteristics, and referral. Gut 2000; 46: 78-82.

28. Thompson WG, Longstreth GF, Drossman DA, Heaton KW, IRvine EJ, MüLER-LissNer SA. Functional bowel disorders and functional abdominal pain. Gut 1999; 45 Suppl 2: II43-7.

29. Longstreth GF, THompson WG, Chey WD, Houghton LA, MeArin F, Spiuer RC. Functional bowel disorders. Gastroenterology 2006; 130: 1480-91.

30. Gerson C, Gerson MJ, Awad RA, Chowdhury A, Dancey C, Poitras P ET al. Irritable bowel síndrome: an internacional study of symptoms in eight countries. Eur J Gastroenterol Hepatol 2008; 20: 659-67.

31. Drossman DA, Weiniand SR. Commentary: sociocultural factors in medicine and gastrointestinal research. Eur J Gastroenterol Hepatol 2008; 20: 593-5. 